\title{
Centrohermitian Solutions of a Factorization Problem Arising in Vibration Analysis. Part II: A Coninvolutory Matrix Approach
}

\author{
Elisa Hubert ${ }^{1}$, Yacine Bouzidi ${ }^{2}$, Roudy Dagher ${ }^{3}$ and Alban Quadrat ${ }^{4}$
}

\begin{abstract}
In [13], we showed that the structure of centrohermitian matrices and Lee's transformation can be used to transform the search for centrohermitian solutions of a rank factorization problem - at the core of a new demodulation approach arising in gearbox vibration analysis - into the search for real solutions of a polynomial system. Hence, in [13], we parametrized a class of centrohermitian solutions of the rank factorization problem that is interesting in practice. Despite its effectiveness, Lee's transformation can be seen as a black box hiding information on the resolution of the rank factorization problem for centrohermitian solutions. To get more insight, in this paper, we develop an alternative approach to the centrohermitian rank factorization problem.
\end{abstract}

\section{INTRODUCTION}

In [13], we studied the centrohermitian solutions of a rank factorization problem defined by centrohermitian matrices [14], [5]. Recall that a matrix with complex entries is centrohermitian when it is equal to the matrix obtained by exchanging the rows and the columns of its conjugate. See Definition 1 below. Centrohermitian vectors and matrices usually appear in signal processing. For instance, the vectors $n \in \mathbb{N}, C_{n}:=\left(c_{-n}(s) \ldots c_{-1}(s) c_{0}(s) c_{1}(s) \ldots c_{n}(s)\right)^{T}$ formed by the $n$ first Fourier coefficients centered around 0 of a real signal $s$ are centrohermitian since $J_{n} \overline{C_{n}}=C_{n}$, where $J_{n}$ denotes the $n \times n$ exchange matrix, i.e., the matrix formed by 1 on the second diagonal and 0 elsewhere.

The rank factorization problem studied in [13] is at the core of demodulation problems arising in gearbox vibration analysis [8], [9]. Indeed, the static transmission error of toothed gearing vibration can be expressed as a finite sum of products of time functions [15], [1], [2], [8]. If we study this error within the frequency domain using Fourier analysis, transforming products into convolutions, under non overlapping hypotheses on the supports of the corresponding signals, we are then led to the rank factorization problem studied in [8], [9], [10], [11], [12], [13]. The standard amplitude and amplitude and phase demodulation problems can be reformulated within this framework [8], [9].

In [13], using the centrohermitian structure and the socalled Lee's transformation [14], [5], the search of centrohermitian solutions of the rank factorization problem was transformed into the search of real solutions of an associated

\footnotetext{
${ }^{1}$ Elisa Hubert is with University of Lyon, UJM-St-Etienne, LASPI, 42334 Saint-Etienne, France elisa.hubert @ wanadoo.fr

${ }^{2}$ Yacine Bouzidi bouzidi.yacinelgmail.com

${ }^{3}$ Roudy Dagher is with Inria Lille - Nord Europe, 59650 Villeneuve d'Ascq, France roudy.dagher@inria.fr

${ }^{4}$ Alban Quadrat is with Inria Paris, Ouragan project, IMJ - PRG, Sorbonne University, 75252 Paris, France alban. quadrateinria.fr
}

rank factorization problem for real matrices. Then, using results obtained in [11], [12], an explicit characterization of a class of centrohermitian solutions (important in practice) of the rank factorization problem was obtained in [13].

Despite its effectiveness, Lee's transformation can be seen as a black box which hides information on the resolution of the rank factorization problem. Hence, in this paper, we shall directly study the existence and the characterization of a class of centrohermitian solutions of the rank factorization problem by adapting the method developed in [10], [11], [12], [13]. Doing so, we shall obtain more insight.

\section{THE CENTROHERMITIAN RANK FACTORIZATION PROBLEM}

In what follows, $\mathbb{k}$ denotes a field (e.g., $\mathbb{Q}, \mathbb{R}, \mathbb{C}$ ), $\mathbb{k}^{n \times m}$ the $\mathbb{k}$-vector space of the $n \times m$ matrices with entries in $\mathbb{k}$, and $I_{n}$ the identity matrix of $\mathbb{k}^{n \times n}$. Moreover, $\bar{M} \in \mathbb{C}^{n \times m}$ (resp., $M^{\star} \in \mathbb{C}^{m \times n}$ ) denotes the conjugate matrix (resp., the adjoint, i.e., the conjugate transpose) of $M \in \mathbb{C}^{n \times m}$ and $J_{n}$ stands for the $n \times n$ exchange matrix, i.e., the matrix formed by 1 on the second diagonal (anti-diagonal) and 0 elsewhere. If $M \in \mathbb{k}^{n \times m}$, then we can consider the $\mathbb{k}$-linear maps $M .: \mathbb{k}^{m \times 1} \longrightarrow \mathbb{k}^{n \times 1}$ and $. M: \mathbb{k}^{1 \times n} \longrightarrow \mathbb{k}^{1 \times m}$ respectively defined by $(M).(\eta):=M \eta$ for all $\eta \in \mathbb{k}^{m \times 1}$ and $(. M)(\lambda):=\lambda M$ for all $\lambda \in \mathbb{K}^{1 \times n}$, and denote their kernels (resp., images) respectively by $\operatorname{ker}_{\mathbb{k}}(M$.$) and$ $\operatorname{ker}_{\mathbb{k}}(. M)$ (resp., $\operatorname{im}_{\mathbb{k}}(M$.$\left.) and \operatorname{im}_{\mathbb{k}}(. M)\right)$. If $\left\{f_{j}\right\}_{j=1, \ldots, r}$ is a set of vectors, then $\operatorname{span}_{\mathbb{k}}\left\{f_{j}\right\}_{j=1, \ldots, r}$ denotes the $\mathbb{k}$-vector generated by the $f_{j}$ 's. Finally, we set:

$$
r \geq l, \quad C_{r}^{l}:=\frac{r !}{l !(r-l) !} .
$$

We can now state the main problem studied in this paper. The rank factorization problem:

Let $D_{1}, \ldots, D_{r} \in \mathbb{k}^{n \times n} \backslash\{0\}$ and $M \in \mathbb{k}^{n \times m} \backslash\{0\}$. Determine - when they exist - a column vector $u \in \mathbb{k}^{n \times 1}$ and row vectors $v_{i} \in \mathbb{k}^{1 \times m}, i=1, \ldots, r$, satisfying:

$$
M=\sum_{i=1}^{r} D_{i} u v_{i}
$$

If (1) has a solution, then $M w=\sum_{i=1}^{r} D_{i} u\left(v_{i} w\right)$ for all $w \in \mathbb{k}^{m \times 1}$ and $\operatorname{im}_{\mathbb{k}}(M.) \subseteq \operatorname{span}_{\mathbb{k}}\left\{D_{1} u, \ldots, D_{r} u\right\} \subseteq$ $\mathbb{k}^{n \times 1}$, which yields the following rank condition:

$$
l:=\operatorname{rank}_{\mathbb{k}}(M) \leq \operatorname{rank}_{\mathbb{k}}\left(D_{1} u \ldots D_{r} u\right) \leq \min \{r, n\} .
$$

Hence, (2) is a necessary condition for the solvability of (1).

Let us state again the concept of a centrohermitian matrix. 
Definition 1 ([14], [5]): A matrix $M \in \mathbb{C}^{n \times m}$ is called centrohermitian if $J_{n} \bar{M} J_{m}=M$. The set of all the centrohermitian matrices of $\mathbb{C}^{n \times m}$ is denoted by $\mathrm{CH}_{n, m}$.

Example 1: Using $J_{1}=1$, a column vector $u \in \mathbb{C}^{n \times 1}$ and a row vector $v_{i} \in \mathbb{C}^{1 \times m}$ are centrohermitian if we have:

$$
J_{n} \bar{u}=u, \quad \overline{v_{i}} J_{m}=v_{i} .
$$

The centrohermitian rank factorization problem is a rank factorization problem for centrohermitian matrices $M$ and $D_{1}, \ldots, D_{r}$ and centrohermitian solutions $u$ and $v_{1}, \ldots, v_{r}$.

The centrohermitian rank factorization problem:

Let $D_{1}, \ldots, D_{r} \in \mathrm{CH}_{n, n} \backslash\{0\}$ and $M \in \mathrm{CH}_{n, m} \backslash\{0\}$. Determine - when they exist $-u \in \mathrm{CH}_{n, 1}$ and $v_{i} \in \mathrm{CH}_{1, m}$, $i=1, \ldots, r$, satisfying $(1)$.

In [13], we showed that the centrohermitian rank factorization problem can be reduced to a rank factorization problem over $\mathbb{k}=\mathbb{R}$. Let us state again this important result.

Definition 2: A matrix $Q \in \mathbb{C}^{n \times m}$ is called $J$-real if:

$$
J_{n} \bar{Q}=Q .
$$

According to Example 1, the columns of a $J$-real matrix are centrohermitian vectors. Let us give explicit examples.

Example 2: For $t \in \mathbb{N}$, the following matrices

$$
\begin{aligned}
Q_{2 t} & =\frac{1}{\sqrt{2}}\left(\begin{array}{cc}
I_{t} & i I_{t} \\
J_{t} & -i J_{t}
\end{array}\right) \in \mathbb{C}^{2 t \times 2 t}, \quad Q_{1}=1, \\
Q_{2 t+1} & =\frac{1}{\sqrt{2}}\left(\begin{array}{ccc}
I_{t} & 0 & i I_{t} \\
0 & \sqrt{2} & 0 \\
J_{t} & 0 & -i J_{t}
\end{array}\right) \in \mathbb{C}^{(2 t+1) \times(2 t+1)}
\end{aligned}
$$

are $J$-real. They are also unitary, namely, they satisfy:

$$
\begin{aligned}
Q_{2 t}^{\star} Q_{2 t} & =Q_{2 t} Q_{2 t}^{\star}=I_{2 t}, \\
Q_{2 t+1}^{\star} Q_{2 t+1} & =Q_{2 t+1} Q_{2 t+1}^{\star}=I_{2 t+1} .
\end{aligned}
$$

If $U \in \mathbb{C}^{m \times m}$ and $V \in \mathbb{C}^{n \times n}$ are two non-singular $J$-real matrices, then we can define Lee's transformation [14]:

$$
\begin{aligned}
\varphi: \mathrm{CH}_{n, m} & \longrightarrow \mathbb{R}^{n \times m} \\
M & \longmapsto V^{-1} M U .
\end{aligned}
$$

In [14], it is shown that $\varphi$ is a bijection, and thus, that the $\mathbb{R}$ vector space $\mathrm{CH}_{n, m}=\varphi^{-1}\left(\mathbb{R}^{n \times m}\right)$ is isomorphic to $\mathbb{R}^{n \times m}$.

We state again the equivalence between the two problems.

Theorem 1 ([13]): Let $D_{i} \in \mathrm{CH}_{n, n}$ for $i=1, \ldots, r$, $M \in \mathrm{CH}_{n, m}, Q_{m} \in \mathbb{C}^{m \times m}$ and $Q_{n} \in \mathbb{C}^{n \times n}$ be two $J$ real and unitary matrices (see, e.g., Example 2), and:

$$
\left\{\begin{array}{l}
D_{i \varphi}:=Q_{n}^{\star} D_{i} Q_{n} \in \mathbb{R}^{n \times n}, \quad i=1, \ldots, r, \\
M_{\varphi}:=Q_{n}^{\star} M Q_{m} \in \mathbb{R}^{n \times m} .
\end{array}\right.
$$

Then, the centrohermitian rank factorization problem (1) admits a solution $\left(u, v_{1}, \ldots, v_{r}\right) \in \mathrm{CH}_{n, 1} \times \mathrm{CH}_{1, m}^{r}$ iff the following rank factorization problem

$$
M_{\varphi}=\sum_{i=1}^{r} D_{i \varphi} u_{\varphi} v_{i \varphi}
$$

admits a solution $\left(u_{\varphi}, v_{1 \varphi}, \ldots, v_{r \varphi}\right) \in \mathbb{R}^{n \times 1} \times\left(\mathbb{R}^{1 \times m}\right)^{r}$. The bijection between the sets of solutions is then defined by:

$$
\begin{aligned}
\mathrm{CH}_{n, 1} \times \mathrm{CH}_{1, m}^{r} & \longmapsto \mathbb{R}^{n \times 1} \times\left(\mathbb{R}^{1 \times m}\right)^{r} \\
\left(u, v_{1}, \ldots, v_{r}\right) & \longmapsto\left(Q_{n}^{\star} u, v_{1} Q_{m}, \ldots, v_{r} Q_{m}\right),
\end{aligned}
$$

$$
\begin{aligned}
\mathbb{R}^{n \times 1} \times\left(\mathbb{R}^{1 \times m}\right)^{r} & \longmapsto \mathrm{CH}_{n, 1} \times \mathrm{CH}_{1, m}^{r} \\
\left(u_{\varphi}, v_{1 \varphi}, \ldots, v_{r \varphi}\right) & \longmapsto\left(Q_{n} u_{\varphi}, v_{1 \varphi} Q_{m}^{\star}, \ldots, v_{r \varphi} Q_{m}^{\star}\right) .
\end{aligned}
$$

III. A FEW INTRODUCING REMARKS

Based on results of [10], [11], [12], all the centrohermitian solutions of the centrohermitian rank factorization problem which are such that $v_{1}, \ldots, v_{r}$ are $\mathbb{C}$-linearly independent were parametrized in [13]. This class is important in practice.

Lee's transformation can be seen as a black box hiding interesting information on the resolution of the centrohermitian rank factorization problem. Hence, we shall adapt the approach developed in [10], [11], [12] to directly handle the centrohermitian rank factorization problem.

If we note

$A(u):=\left(\begin{array}{llll}D_{1} u & \ldots & D_{r} u\end{array}\right) \in \mathbb{k}^{n \times r}, v:=\left(\begin{array}{c}v_{1} \\ \vdots \\ v_{r}\end{array}\right) \in \mathbb{k}^{r \times m}$,

then (1) can be written as follows

$$
A(u) v=M,
$$

which emphasizes the bilinear structure of the system (1) which is polynomial in the entries of $u$ and of the $v_{i}$ 's.

Note that (5) and (2) explain the choice of the terminology for the problem (1), namely, a rank factorization problem.

If $(u, v)$ is a solution of (5), then so is $\left(\lambda u, \lambda^{-1} v\right)$ for all $\lambda \in \mathbb{k} \backslash\{0\}$, which shows that the solution space $\mathcal{S}$ of (5) is closed under the following transformation:

$$
\forall \lambda \in \mathbb{k} \backslash\{0\}, \lambda \bullet(u, v):=\left(\lambda u, \lambda^{-1} v\right) .
$$

Since $\mathbb{k}^{\times}:=\mathbb{k} \backslash\{0\}$ is an abelian group for the multiplication, (6) then defines a group action of $\mathbb{k}^{\times}$on $\mathcal{S}$, namely:

$$
\begin{aligned}
\forall(u, v) \in \mathcal{S}, & 1 \bullet(u, v)=(u, v), \\
\forall \lambda_{1}, \lambda_{2} \in \mathbb{R}^{\times}, & \lambda_{2} \bullet\left(\lambda_{1} \bullet(u, v)\right)=\left(\lambda_{2} \lambda_{1}\right) \bullet(u, v) .
\end{aligned}
$$

The action (6) of the multiplicative group $\mathbb{k}^{\times}$on the solution space $\mathcal{S}$ of (5) yields the following equivalence relation:

$$
\begin{gathered}
\forall\left(u^{\prime}, v^{\prime}\right),(u, v) \in \mathcal{S}:\left(u^{\prime}, v^{\prime}\right) \sim(u, v) \\
\Leftrightarrow \exists \lambda \in \mathbb{k}^{\times}:\left(u^{\prime}, v^{\prime}\right)=\lambda \bullet(u, v)=\left(u \lambda, \lambda^{-1} v\right) .
\end{gathered}
$$

The quotient of the action $\mathcal{S} / \mathbb{k}^{\times}$, defined by the orbits

$$
\forall(u, v) \in \mathcal{S}: \mathcal{O}_{(u, v)}:=\left\{\left(u \lambda, \lambda^{-1} v\right) \mid \lambda \in \mathbb{k}^{\times}\right\},
$$

defines a partition of the solution space $\mathcal{S}$ of (5). Hence, the study of $\mathcal{S}$ can be restricted to the study of $\mathcal{S} / \mathbb{k}^{\times}$.

In what follows, we shall suppose that $\mathbb{k}=\mathbb{C}$.

Let us first suppose that a centrohermitian solution $(u, v)$ exists for (1), i.e., $(u, v)$ satisfies (5) and:

$$
\begin{aligned}
u & =J_{n} \bar{u}, \quad v_{i}=\overline{v_{i}} J_{m}, \quad i=1, \ldots, r, \\
\Leftrightarrow \quad u & =J_{n} \bar{u}, \quad v=\bar{v} J_{m} .
\end{aligned}
$$

Let us suppose that the matrices $D_{i}$ 's are also centrohermitian. Then, we can first check that we have:

$$
\begin{aligned}
\overline{A(u)} & =\left(\bar{D}_{1} \bar{u} \ldots \bar{D}_{r} \bar{u}\right) \\
& =\left(J_{n} D_{1} J_{n} \bar{u} \ldots J_{n} D_{r} J_{n} \bar{u}\right) \\
& =J_{n} A\left(J_{n} \bar{u}\right) .
\end{aligned}
$$


Using (7) and (8), $\overline{A(u)} \bar{v}=\bar{M}$ yields $J_{n} A(u) v J_{m}=\bar{M}$. Then, we obtain $A(u) v=J_{n} \bar{M} J_{m}$, i.e., $J_{n} \bar{M} J_{m}=M$. Hence, $M$ is necessarily a centrohermitian matrix.

Let $M, D_{1}, \ldots, D_{r}$ be centrohermitian matrices. Let us study when a complex solution $(u, v)$ of (5) is a centrohermitian solution, i.e., satisfies (7). Using (5), we get $\overline{A(u)} \bar{v}=\bar{M}=J_{n} M J_{m}$, and thus, using (8), we have $J_{n} \overline{A(u)} \bar{v} J_{m}=A\left(J_{n} \bar{u}\right)\left(\bar{v} J_{m}\right)=M$. The set $\mathcal{S}$ of complex solutions of (5) is then closed under the transformation:

$$
\mathcal{T}:(u, v) \in \mathcal{S} \longmapsto\left(J_{n} \bar{u}, \bar{v} J_{m}\right) \in \mathcal{S} .
$$

The transformation $\mathcal{T}$ satisfies $\mathcal{T}^{2}=\mathrm{id}$, where id is the identity transformation. Moreover, using (6), we obtain:

$$
\begin{aligned}
\forall \lambda \in \mathbb{k}^{\times}, \quad \mathcal{T}(\lambda \bullet(u, v)) & =\mathcal{T}\left(u \lambda, \lambda^{-1} v\right) \\
& =\left(J_{n} \bar{u} \bar{\lambda}, \bar{\lambda}^{-1} \bar{v} J_{m}\right) \\
& =\bar{\lambda} \bullet \mathcal{T}(u, v) .
\end{aligned}
$$

Hence, we can restrict $\mathcal{T}$ to the quotient $\mathcal{S} / \mathbb{k}^{\times}$to obtain:

$$
\begin{aligned}
\overline{\mathcal{T}}: \mathcal{S} / \mathbb{k}^{\times} & \longrightarrow \mathcal{S} / \mathbb{k}^{\times} \\
\mathcal{O}_{(u, v)} & \longmapsto \mathcal{O}_{\mathcal{T}(u, v)} .
\end{aligned}
$$

The centrohermitian solutions of (5), i.e., the solutions satisfying (7), are the fixed-points of $\mathcal{T}$, i.e., those that satisfy:

$$
(u, v) \in \mathcal{S}: \mathcal{T}(u, v)=(u, v) .
$$

Lemma 1: The centrohermitian solutions of (5) are in a 1-1 correspondence with the orbits $\mathcal{O}_{(u, v)}$ of $\mathcal{S} / \mathbb{k}^{\times}$that are fixed by the involution $\overline{\mathcal{T}}$ defined by (10).

Proof: If $(u, v)$ is a centrohermitian solution of (5), i.e., if $(u, v)$ is a fixed point of $\mathcal{T}$, then, using (10), we have $\overline{\mathcal{T}}\left(\mathcal{O}_{(u, v)}\right)=\mathcal{O}_{\mathcal{T}(u, v)}=\mathcal{O}_{(u, v)}$, which shows that the orbit $\mathcal{O}_{(u, v)}$ is fixed by $\overline{\mathcal{T}}$. Conversely, let us suppose that $\mathcal{O}_{(u, v)}$ is a fixed point of $\overline{\mathcal{T}}$, then $\mathcal{O}_{\mathcal{T}(u, v)}=\mathcal{O}_{(u, v)}$. By definition, there exists $z \in \mathbb{k}^{\times}$such that $\mathcal{T}(u, v)=\left(z u, z^{-1} v\right)$, i.e., $\left(J_{n} \bar{u}, \bar{v} J_{m}\right)=\left(z u, z^{-1} v\right)$. The equation $J_{n} \bar{u}=z u$ yields $u=J_{n} \overline{z u}=\bar{z} J_{n} \bar{u}=\bar{z} z u$. Since $u \neq 0(u=0$ yields $A(u)=0$, and thus, $M=0$ ), we obtain $z \bar{z}=|z|^{2}=1$. Let us first suppose that $z=-1$ and let $u^{\prime}=i u$ and $v^{\prime}=-i v$. Then, we have $\mathcal{O}_{\left(u^{\prime}, v^{\prime}\right)}=\mathcal{O}_{(u, v)}$,

$$
\left\{\begin{array}{l}
J_{n} \overline{u^{\prime}}=-i J_{n} \bar{u}=i u=u^{\prime}, \\
\overline{v^{\prime}} J_{m}=i \bar{v} J_{m}=-i v=v^{\prime}
\end{array}\right.
$$

which shows that $\left(u^{\prime}, v^{\prime}\right)$ is a centrohermitian solution. Now, let us suppose that $z \neq-1$ and let $\lambda:=(1+z) / 2 \in \mathbb{k}^{\times}$, $u^{\prime}:=\lambda u$ and $v^{\prime}:=\lambda^{-1} v$. Then, we have $\mathcal{O}_{\left(u^{\prime}, v^{\prime}\right)}=\mathcal{O}_{(u, v)}$, $\bar{\lambda} z=\lambda$, and thus, $\bar{\lambda}^{-1} z^{-1}=\lambda^{-1}$ and

$$
\left\{\begin{array}{l}
J_{n} \overline{u^{\prime}}=\bar{\lambda} J_{n} \bar{u}=\bar{\lambda} z u=\lambda u=u^{\prime}, \\
\overline{v^{\prime}} J_{m}=\bar{\lambda}^{-1} \bar{v} J_{m}=\bar{\lambda}^{-1} z^{-1} v=\lambda^{-1} v=v^{\prime},
\end{array}\right.
$$

which shows that $\left(u^{\prime}, v^{\prime}\right)$ is a centrohermitian solution.

Remark 1: If $\left(u_{J}, v\right)$ satisfies (5), where $u_{J}$ is centrohermitian, i.e., $J_{n} \overline{u_{J}}=u_{J}$, then, using (9), we have $A\left(u_{J}\right)\left(\bar{v} J_{m}\right)=M$, which, with the following notation

$$
v_{J}:=\frac{1}{2}\left(v+\bar{v} J_{m}\right)
$$

yields $A\left(u_{J}\right) v_{J}=M$ and $\overline{v_{J}} J_{m}=v_{J}$, and shows that $\left(u_{J}, v_{J}\right)$ is a centrohermitian solution of (5). Hence, the problem of the existence of fixed-points of $\mathcal{T}$ is reduced to the problem of the existence of solutions $(u, v)$ of (5) satisfying $J_{n} \bar{u}=u$, i.e., of solutions with $u$ centrohermitian.

\section{Characterization OF A ClAss OF SOlutions}

In Section IV-A below, we shall review the parametrization of all the solutions $(u, v)$ of (5) which are such that $v$ are full row rank matrices which is obtained in [10], [11], [12]. These solutions are important in practice [8]. In this paper, we shall focus on this important class of solutions. See [3] for the general solutions. In Section IV-B, we shall extend this parametrization for the centrohermitian rank factorization problem without using Lee's transformations (see Section II).

\section{A. Full row rank solutions $v$}

(5) is equivalent to the existence of $u \in \mathbb{k}^{n \times 1}$ satisfying:

$$
\operatorname{im}_{\mathbb{k}}(M .) \subseteq \operatorname{im}_{\mathbb{k}}(A(u) .) \subseteq \mathbb{k}^{n \times 1} .
$$

Thus, we find again (2), i.e.:

$$
l:=\operatorname{rank}_{\mathbb{k}}(M) \leq \operatorname{rank}_{\mathbb{k}}(A(u) .) \leq \min \{r, n\} .
$$

Hence, $u \in \mathbb{k}^{n \times 1}$ is such that not all the $l \times l$-minors of the matrix $A(u)$ vanish simultaneously.

A class of solutions of (5), important in practice, is defined by the solutions $(u, v)$ with full row rank matrices $v$, i.e., such that the $v_{i}$ 's are $\mathbb{k}$-linearly independent. Note that $v$ then admits a right inverse $t \in \mathbb{k}^{m \times r}$, i.e., $v t=I_{r}$. Hence, $A(u) v=M$ yields $A(u)=M t$, which shows the inclusion $\operatorname{im}_{\mathbb{k}}(A(u).) \subseteq \operatorname{im}_{\mathbb{k}}(M$.$) and with (11) yields:$

$$
\operatorname{im}_{\mathbb{k}}(A(u) .)=\operatorname{im}_{\mathbb{k}}\left(D_{1} u \ldots D_{r} u\right)=\operatorname{im}_{\mathbb{k}}(M .) .
$$

Remark 2: Alternatively, this result is a consequence of the fact that $\operatorname{rank}_{\mathbb{k}}(M)=\operatorname{rank}_{\mathbb{k}}(A(u) v)=\operatorname{rank}_{\mathbb{k}}(A(u))$ since $\operatorname{rank}_{\mathbb{k}}(v)=r$, i.e., of the fact that $v$ has full row rank. Equivalently, it is also a straightforward consequence of (12) and of Sylvester's rank inequality asserting that:

$$
\operatorname{rank}_{\mathbb{k}}(A(u))+\operatorname{rank}_{\mathbb{k}}(v) \leq l+r .
$$

Indeed, $\operatorname{rank}_{\mathbb{k}}(v)=r$ and (12) yield $l \leq \operatorname{rank}_{\mathbb{k}}(A(u)) \leq l$.

In what follows, we shall recall how to parametrize all the $u$ 's satisfying the equality (13). Note (13) is equivalent to:

1) $D_{i} u \in \operatorname{im}_{\mathbb{k}}(M)=.\operatorname{ker}_{\mathbb{k}}(L$.$) for i=1, \ldots, r$, where $L \in \mathbb{k}^{p \times n}$ is a full row rank matrix whose rows define a basis of $\operatorname{ker}_{\mathbb{k}}(. M)$ and $p:=n-l$, i.e., $\operatorname{ker}_{\mathbb{k}}(. M)=\operatorname{im}_{\mathbb{k}}(L$.$) (with the convention that L=0$ if $\operatorname{ker}_{\mathbb{k}}(. M)=0$ ), i.e., $u$ satisfies the linear system:

$$
N u=0, \quad N:=\left(\begin{array}{c}
L D_{1} \\
\vdots \\
L D_{r}
\end{array}\right) \in \mathbb{k}^{p r \times n} .
$$

2) $\operatorname{rank}_{\mathbb{k}}(A(u))=.\operatorname{rank}_{\mathbb{k}}(M)=l$.

These above two conditions can be solved as follows. If (14) is reduced to $\{0\}$, then no solution exists since $A(0)=0$ and $M \neq 0$. Hence, let us suppose that the dimension $d$ of the 
k-vector space (14), i.e., of $\operatorname{ker}_{\mathbb{k}}(N$.$) , is larger than or equal$ to 1 and let $Z \in \mathbb{k}^{n \times d}$ be a full column rank matrix whose columns define a basis of (14). Thus, $u=Z \psi$ satisfies (14) for all $\psi \in \mathbb{k}^{d \times 1}$, i.e., satisfies the first above condition. Substituting this expression into the second condition, we are led to the characterization of the following set:

$$
\mathcal{P}:=\left\{\psi \in \mathbb{k}^{d \times 1} \mid \operatorname{rank}_{\mathbb{k}}(A(Z \psi))=l\right\} .
$$

To do that, let $X \in \mathbb{k}^{n \times l}$ be a full column rank matrix whose columns define a basis of $\operatorname{im}_{\mathbb{k}}(M$.). Therefore, we have $\operatorname{im}_{\mathbb{k}}(M)=.\operatorname{im}_{\mathbb{k}}(X$.$) and there exists a unique matrix$ $Y \in \mathbb{k}^{l \times m}$ such that $M=X Y$. Now, using the fact that $D_{i} Z \psi \in \operatorname{im}_{\mathbb{k}}(M)=.\operatorname{im}_{\mathbb{k}}\left(X\right.$.) for all $\psi \in \mathbb{k}^{d \times 1}$, there exists a unique $W_{i} \in \mathbb{k}^{l \times d}$ such that $D_{i} Z=X W_{i}$ for $i=1, \ldots, r$, which yields $A(Z \psi)=X\left(W_{1} \psi \ldots W_{r} \psi\right)$ for all $\psi \in \mathbb{k}^{d \times 1}$. Let $B(\psi):=\left(W_{1} \psi \ldots W_{r} \psi\right) \in \mathbb{k}^{l \times r}$ for $\psi \in \mathbb{k}^{d \times 1}$. Since $X$ has full column rank, we then get:

$$
\mathcal{P}=\left\{\psi \in \mathbb{k}^{d \times 1} \mid \operatorname{rank}_{\mathbb{k}}(B(\psi))=l\right\} .
$$

Let $\left\{\mathfrak{m}_{i}\right\}_{i=1, \ldots, C_{r}^{l}}$ denote the set of all the $l \times l$-minors of the matrix $B(\psi)^{r} \in \mathbb{k}^{l \times r}$. Note that $\mathfrak{m}_{i}$ is either 0 or a homogeneous polynomial of degree $l$. Let $\left\langle\mathfrak{m}_{1}, \ldots, \mathfrak{m}_{C_{r}^{l}}\right\rangle$ be the ideal of the polynomial ring $\mathbb{k}\left[\psi_{1}, \ldots, \psi_{d}\right]$ and let us consider the following affine algebraic set [4]:

$$
\begin{gathered}
V_{\mathbb{k}}\left(\left\langle\mathfrak{m}_{1}, \ldots, \mathfrak{m}_{C_{r}^{l}}\right\rangle\right):= \\
\left\{\psi=\left(\psi_{1} \ldots \psi_{r}\right)^{T} \in \mathbb{k}^{d \times 1} \mid \mathfrak{m}_{i}(\psi)=0, i=1, \ldots, C_{r}^{l}\right\} .
\end{gathered}
$$

Then, (15) is clearly equivalent to:

$$
\mathcal{P}=\mathbb{k}^{d \times 1} \backslash V_{\mathbb{k}}\left(\left\langle\mathfrak{m}_{1}, \ldots, \mathfrak{m}_{C_{r}^{l}}\right\rangle\right) .
$$

For a more efficient method to compute $\mathcal{P}$, see [12].

For every $\psi \in \mathcal{P}, B(\psi)$ has full row rank, and thus, there exists a right inverse $E_{\psi} \in \mathbb{k}^{r \times l}$, i.e., $B(\psi) E_{\psi}=I_{r}$. Using $X B(\psi) v=A(Z \psi) v=M=X Y$ and the fact that $X$ has full column rank, we obtain $B(\psi) v=Y$, which yields $v=$ $E_{\psi} Y+C_{\psi} Y^{\prime}$, where $C_{\psi} \in \mathbb{k}^{r \times(r-l)}$ is a full column rank matrix whose columns define a basis of the $\mathbb{k}$-vector space $\operatorname{ker}_{\mathbb{k}}\left(B(\psi)\right.$.), i.e., $\operatorname{ker}_{\mathbb{k}}\left(B(\psi)\right.$.) $=\operatorname{im}_{\mathbb{k}}\left(C_{\psi}\right)$, and $Y^{\prime}$ is any matrix of $\mathbb{k}^{(r-l) \times m}$. Finally, noticing that $\left(E_{\psi} \quad C_{\psi}\right) \in \mathbb{k}^{r \times r}$ is not singular, $v$ has full row rank iff so has $\left(Y^{T} Y^{\prime T}\right)$.

Theorem 2 ([11]): With the above notations, we have:

$\forall \psi \in \mathcal{P}, \forall Y^{\prime} \in \mathbb{k}^{(r-l) \times m},\left(\begin{array}{c}u=Z \psi \\ v=E_{\psi} Y+C_{\psi} Y^{\prime}\end{array}\right) \in \mathcal{S}$.

Moreover, $v=E_{\psi} Y+C_{\psi} Y^{\prime}$ has full row rank iff so has

$$
\left(\begin{array}{ll}
Y^{T} & Y^{\prime T}
\end{array}\right) \text {. }
$$

See [3], [12] for the study of the (regularity of the) maps:

$$
\psi \in \mathcal{P} \longmapsto E_{\psi}, \quad \psi \in \mathcal{P} \longmapsto C_{\psi} .
$$

Note that the multiplicative group $\mathbb{k}^{\times}$acts on $\mathcal{P}$ as follows:

$$
\begin{aligned}
\mathbb{k}^{\times} \times \mathcal{P} & \longrightarrow \mathcal{P} \\
(\lambda, \psi) & \longmapsto \lambda \psi .
\end{aligned}
$$

Hence, the study of $\mathcal{P}$ can be reduced to the study of the quotient of the action $\mathcal{P} / \mathbb{k}^{\times}$and of the orbits $\mathcal{O}_{\psi}$ for $\psi \in \mathcal{P}$.
Moreover, we note that $B(\lambda \psi)=\lambda B(\psi)$ for all $\lambda \in \mathbb{k}^{\times}$. Hence, we get that $C_{\lambda \psi}=C_{\psi}$. If we denote by $E_{\lambda \psi}$ a right inverse of $B(\lambda \psi)$ for $\psi \in \mathcal{P}$, i.e., $B(\lambda \psi) E_{\lambda \psi}=I_{r}$, then we get $B(\psi)\left(\lambda E_{\lambda \psi}\right)=I_{r}$, and thus, $B(\psi)\left(E_{\psi}-\lambda E_{\lambda \psi}\right)=0$, which shows that each column of $E_{\psi}-\lambda E_{\lambda \psi}$ belongs to $\operatorname{ker}_{\mathbb{k}}\left(B(\psi)\right.$.) $=\operatorname{im}_{\mathbb{k}}\left(C_{\psi}.\right)$, and thus, there exists a unique matrix $Y_{\psi}^{\prime \prime} \in \mathbb{k}^{(r-l) \times l}$ such that $E_{\psi}-\lambda E_{\lambda \psi}=C_{\psi} Y_{\psi}^{\prime \prime}$. Hence, we have:

$$
\lambda^{-1} E_{\psi}=E_{\lambda \psi}-\lambda^{-1} C_{\psi} Y_{\psi}^{\prime \prime}=E_{\lambda \psi}-\lambda^{-1} C_{\lambda \psi} Y_{\psi}^{\prime \prime} .
$$

Finally, if $(u, v) \in \mathcal{S}$ is of the form of (16), then we have:

$$
\begin{gathered}
\forall \lambda \in \mathbb{k}^{\times}, \lambda \bullet(u, v)=\left(\lambda u, \lambda^{-1} v\right) \\
=\left(Z \lambda \psi, E_{\lambda \psi} Y+C_{\lambda \psi} \lambda^{-1}\left(Y^{\prime}-Y_{\psi}^{\prime \prime} Y\right)\right) \in \mathcal{S} .
\end{gathered}
$$

For examples, see [3], [8], [9], [10], [11], [12], [13].

\section{B. An approach based on coninvolutory matrices}

We consider the extension of the approach for the rank factorization problem of Section IV-A to the centrohermitian rank factorization problem, i.e., to centrohermitian matrices $M, D_{1}, \ldots, D_{r}$ and centrohermitian solutions $u, v_{1}, \ldots, v_{r}$.

Let $\mathbb{k}=\mathbb{C}, M \in \mathrm{CH}_{n, m}$ and $D_{i} \in \mathrm{CH}_{n, n}, i=1, \ldots, r$.

By definition of the full row rank matrix $L \in \mathbb{k}^{p \times n}$ introduced in Section IV-A, it satisfies the following properties:

1) $L M=0$.

2) If $L^{\prime} \in \mathbb{k}^{p^{\prime} \times n}$ is a matrix satisfying $L^{\prime} M=0$, then there exists $L^{\prime \prime} \in \mathbb{k}^{p^{\prime} \times p}$ such that $L^{\prime}=L^{\prime \prime} L$.

The first condition, i.e., $L M=0$ yields $\bar{L} \bar{M}=0$, i.e., $\bar{L} J_{n} M J_{m}=0$ since $\bar{M}=J_{n} M J_{m}$, and thus, we get $\left(\bar{L} J_{n}\right) M=0$. Using the second point, there exists a matrix $L^{\prime \prime} \in \mathbb{k}^{p \times p}$ such that $\bar{L} J_{n}=L^{\prime \prime} L$, i.e.:

$$
\bar{L}=L^{\prime \prime} L J_{n} \text {. }
$$

Using (17), $J_{n} \in \mathbb{R}^{n \times n}$ and $J_{n}^{2}=I_{n}$, we obtain

$$
L=\overline{L^{\prime \prime}} \bar{L} J_{n}=\overline{L^{\prime \prime}} L^{\prime \prime} L J_{n}^{2}=\overline{L^{\prime \prime}} L^{\prime \prime} L,
$$

and using the fact that $L$ has full row rank, we finally obtain:

$$
\overline{L^{\prime \prime}} L^{\prime \prime}=I_{p}, \quad L^{\prime \prime} \overline{L^{\prime \prime}}=I_{p} .
$$

A matrix $L^{\prime \prime} \in \mathbb{k}^{p \times p}$ satisfying (18) is called coninvolutory [6], [7]. Clearly, a coninvolutory matrix is non-singular.

Remark 3: If $\Theta \in \mathbb{R}^{n \times n}$, then $e^{i \Theta}$ is clearly coninvolutory. Theorem 14 of [7] proves the converse result, i.e., every coninvolutory matrix $E$ is of the form of $E=e^{i \Theta}$ for a certain $\Theta \in \mathbb{R}^{n \times n}$. In particular, $\Theta$ can be chosen to be a polynomial in $E$. Finally, there exists a unique matrix $\Theta \in \mathbb{R}^{n \times n}$ satisfying $E=e^{i \Theta}$ with all its eigenvalues in the half-open strip $\{z \in \mathbb{C} \mid-\pi / 2<\Im(z)<\pi / 2\}$.

Let us now consider a solution $u \in \mathbb{k}^{n \times 1}$ of (14), i.e., satisfying $L D_{i} u=0$ for $i=1, \ldots, r$. Then, we have $\bar{L} \overline{D_{i}} \bar{u}=0$. Using $\bar{L}=L^{\prime \prime} L J_{n}, \overline{D_{i}}=J_{n} D_{i} J_{n}, J_{n}^{2}=I_{n}$ and $L^{\prime \prime}$ is a non-singular matrix, we then obtain

$$
L^{\prime \prime} L J_{n}^{2} D_{i} J_{n} \bar{u}=0 \Leftrightarrow L D_{i}\left(J_{n} \bar{u}\right)=0,
$$


which shows that the solution $\operatorname{space} \operatorname{ker}_{\mathbb{k}}(N$.$) of (14) is$ closed under the following transformation:

$$
\begin{aligned}
\mathcal{J}: \operatorname{ker}_{\mathbb{k}}(N .) & \longrightarrow \operatorname{ker}_{\mathbb{k}}(N .), \\
u & \longmapsto J_{n} \bar{u} .
\end{aligned}
$$

Using the $\mathbb{k}$-vector space structure of $\operatorname{ker}_{\mathbb{k}}(N$.) coming from the linear system (14), we then have:

$$
u_{J}:=\frac{1}{2}(u+\mathcal{J}(u))=\frac{1}{2}\left(u+J_{n} \bar{u}\right) \in \operatorname{ker}_{\mathbb{k}}(N .) .
$$

Moreover, $u_{J}$ satisfies $\mathcal{J}\left(u_{J}\right)=u_{J}$, i.e., $u \in \mathrm{CH}_{n, 1}$, which shows (14) always admits centrohermitian solutions.

If $(u, v) \in \mathcal{S}$, then we have just proved that $u_{J} \in \mathrm{CH}_{n, 1}$ satisfies (14). But as explained in Section IV-A, (14) is only a necessary condition on $u$ for the existence of a solution $(u, v)$ of (5). Hence, we now need to investigate when there exists $v_{J} \in \mathbb{k}^{r \times m}$ satisfying $\overline{v_{J}} J_{m}=v_{J}$ (i.e., the rows of $v_{J}$ are centrohermitian vectors) such that $\left(u_{J}, v_{J}\right) \in \mathcal{S}$.

Recall that $u=Z \psi$ is a solution of (14) for all $\psi \in \mathbb{k}^{d \times 1}$. Hence, using (19), we get that $J_{n} \bar{u}=J_{n} \bar{Z} \bar{\psi}$ is also a solution of (14) for all $\psi \in \mathbb{k}^{d \times 1}$. Hence, there exists a unique $\phi \in \mathbb{k}^{d \times 1}$ such that $J_{n} \bar{u}=J_{n} \bar{Z} \bar{\psi}=Z \phi$. Considering $R:=\left(\begin{array}{lll}\phi_{1} & \ldots & \phi_{n}\end{array}\right) \in \mathbb{k}^{d \times d}$, where $\phi_{i}$ is such that $J_{n} \bar{Z} \overline{\psi_{i}}=Z \phi_{i}$ and $\psi_{i}$ is the $i^{\text {th }}$ vector of the standard basis of $\mathbb{k}^{n \times 1}$, i.e., $\psi_{i}$ is the vector formed by 1 at the $i^{\text {th }}$ position and 0 elsewhere, then we have $J_{n} \bar{Z}=Z R$, i.e.:

$$
\bar{Z}=J_{n} Z R \text {. }
$$

Then, we get $Z=J_{n} \bar{Z} \bar{R}$, which implies that $Z=Z R \bar{R}$, and thus, $R \bar{R}=I_{d}$ since $Z$ has full column rank, and also

$$
\bar{R} R=R \bar{R}=I_{d},
$$

i.e., $R$ is coninvolutory. Using $u=Z \psi$ and (20), we have:

$$
J_{n} \bar{u}=J_{n} \bar{Z} \bar{\psi}=Z(R \bar{\psi}) \in \operatorname{ker}_{\mathbb{k}}(N .) .
$$

Now, using (20) and the fact that $Z$ has full row rank, $u=Z \psi$ is centrohermitian iff $J_{n} \bar{u}=u$, i.e., iff:

$$
J_{n} \bar{Z} \bar{\psi}=Z \psi \Leftrightarrow Z(R \bar{\psi}-\psi)=0 \Leftrightarrow \psi=R \bar{\psi} .
$$

A vector $\psi \in \mathbb{k}^{d \times 1}$ satisfying (23) will be called $R$ conjugate. Let $\mathcal{R}$ be the $\mathbb{R}$-linear vector space formed by all the $R$-conjugate vectors of $\mathbb{k}^{d \times 1}$, i.e.:

$$
\mathcal{R}:=\left\{\psi \in \mathbb{k}^{d \times 1} \mid R \bar{\psi}=\psi\right\} .
$$

Hence, $u \in \mathbb{k}^{n \times 1}$ belongs to $\operatorname{ker}_{\mathbb{k}}(N$.$) and is centrohermitian$ iff there exists $\psi \in \mathcal{R}$ such that $u=Z \psi$.

Let $\psi=\psi_{r}+i \psi_{i}$, where $\psi_{r}, \psi_{i} \in \mathbb{R}^{d \times 1}, R=R_{r}+i R_{i}$, where $R_{r}, R_{i} \in \mathbb{R}^{d \times d}$. Using (21), $R_{r}$ and $R_{i}$ satisfy:

$$
R_{r}^{2}+R_{i}^{2}=I_{d}, \quad R_{r} R_{i}=R_{i} R_{r} .
$$

Let us note:

$$
H:=\left(\begin{array}{cc}
R_{r} & R_{i} \\
R_{i} & -R_{r}
\end{array}\right) \in \mathbb{R}^{2 d \times 2 d} .
$$

The matrix $H$ is involutory [6], namely:

$$
H^{2}=\left(\begin{array}{cc}
R_{r} & R_{i} \\
R_{i} & -R_{r}
\end{array}\right)\left(\begin{array}{cc}
R_{r} & R_{i} \\
R_{i} & -R_{r}
\end{array}\right)=I_{2 d} .
$$

Since $H^{2}=I_{2 d}$, the characteristic polynomial of $H$ is either $\lambda^{2}-1$ or one of its two factors, i.e., $\lambda-1, \lambda+1$. The last two correspond to $H=I_{2 d}$ and $H=-I_{2 d}$, which are not of the form of (25). Hence, the characteristic polynomial of $H$ is $\lambda^{2}-1$, which shows that the eigenvalues of $H$ are 1 and -1 associated with the respective eigenvectors of the form:

$$
\eta_{+}:=\eta+H \eta, \quad \eta_{-}:=\eta-H \eta, \quad \eta \in \mathbb{k}^{2 d \times 1} .
$$

Now, $\psi=R \bar{\psi}$ is equivalent to the following linear system

$$
\left(\begin{array}{cc}
R_{r} & R_{i} \\
R_{i} & -R_{r}
\end{array}\right)\left(\begin{array}{c}
\psi_{r} \\
\psi_{i}
\end{array}\right)=\left(\begin{array}{c}
\psi_{r} \\
\psi_{i}
\end{array}\right)
$$

which shows that $\left(\begin{array}{ll}\psi_{r}^{T} & \psi_{i}^{T}\end{array}\right)^{T} \in \mathbb{R}^{2 d \times 1}$ is an eigenvector of $H$ associated with its eigenvalue 1 . Hence, we have $\mathcal{R} \neq 0$.

Computing a basis of the eigenspace $\operatorname{ker}_{\mathbb{R}}\left(\left(H-I_{2 d}\right)\right.$.) of $H$, there exists a full column rank matrix $G \in \mathbb{R}^{2 d \times e}$ such that $\operatorname{ker}_{\mathbb{R}}\left(\left(H-I_{2 d}\right).\right)=\operatorname{im}_{\mathbb{R}}(G$.), where $e$ is the multiplicity of the eigenvalue 1 of $H$, i.e., $e=\operatorname{dim}_{\mathbb{R}}\left(\operatorname{ker}_{\mathbb{R}}\left(\left(H-I_{2 d}\right)\right.\right.$.)). Hence, using $\psi=\psi_{r}+i \psi_{i}$, we have:

$$
\psi=R \bar{\psi} \Leftrightarrow \exists \gamma \in \mathbb{R}^{e \times 1},\left(\begin{array}{c}
\psi_{r} \\
\psi_{i}
\end{array}\right)=G \gamma .
$$

Now, let $G=\left(\begin{array}{ll}G_{1}^{T} & G_{2}^{T}\end{array}\right)^{T}$, where $G_{k} \in \mathbb{R}^{d \times e}$ for $k=1,2$, and let set $0 \neq Z^{\prime}:=G_{1}+i G_{2} \in \mathbb{k}^{d \times e}$. We then have:

$$
\mathcal{R}=Z^{\prime} \mathbb{R}^{e \times 1}=\left\{Z^{\prime} \gamma \mid \gamma \in \mathbb{R}^{e \times 1}\right\} .
$$

Note that, by construction, we have $R \overline{Z^{\prime}}=Z$. This identity can easily be checked again as follows:

$$
H G=G \Leftrightarrow\left\{\begin{array}{l}
R_{r} G_{1}+R_{i} G_{2}=G_{1}, \\
R_{i} G_{1}-R_{r} G_{2}=G_{2},
\end{array} \Leftrightarrow R \overline{Z^{\prime}}=Z^{\prime} .\right.
$$

Using Remark 1 and Theorem 2, we obtain that a solution of the centrohermitian rank factorization problem exists iff there exists $\gamma \in \mathbb{R}^{e \times 1}$ such that the following matrix

$$
K(\gamma):=B\left(Z^{\prime} \gamma\right)=\left(W_{1} Z^{\prime} \gamma \ldots W_{r} Z^{\prime} \gamma\right) \in \mathbb{k}^{l \times r}
$$

is such that $\operatorname{rank}_{\mathbb{k}}(K(\gamma))=l$, i.e., such that $K(\gamma)$ has full row rank, i.e., admits a right inverse.

We can now state the main result of the paper.

Theorem 3: With the above notations, the centrohermitian rank factorization problem admits a solution iff:

$$
\mathcal{C}:=\left\{\gamma \in \mathbb{R}^{e \times 1} \mid \operatorname{rank}_{\mathbb{R}}(K(\gamma))=l\right\} \neq \emptyset .
$$

If so, then centrohermitian solutions are defined by

$$
\forall \gamma \in \mathcal{C}, \quad\left\{\begin{array}{l}
u_{J}=\left(Z Z^{\prime}\right) \gamma \\
v_{J}=\frac{v+\bar{v} J_{m}}{2},
\end{array}\right.
$$

with the following notations:

- $v=E_{\gamma} Y+C_{\gamma} Y^{\prime}$ for all $Y^{\prime} \in \mathbb{k}^{(r-l) \times 1}$,

- $E_{\gamma} \in \mathbb{k}^{r \times l}$ is a right inverse of the matrix $K(\gamma) \in \mathbb{k}^{l \times r}$, 
- $C_{\gamma} \in \mathbb{k}^{r \times(r-l)}$ is a full column rank matrix satisfying:

$$
\operatorname{ker}_{\mathbb{k}}(K(\gamma) .)=\operatorname{im}_{\mathbb{k}}\left(C_{\gamma} .\right) \text {. }
$$

For computational issues, we refer to [3], [12].

Example 3: We consider the centrohermitian matrices:

$$
\begin{gathered}
M=\left(\begin{array}{ccc}
9+18 i & -225 & 9+198 i \\
0 & 0 & 0 \\
9-198 i & -225 & 9-18 i
\end{array}\right), \\
D_{1}=I_{3}, \quad D_{2}=\left(\begin{array}{ccc}
-i & 0 & 0 \\
0 & 0 & 0 \\
0 & 0 & i
\end{array}\right) .
\end{gathered}
$$

$$
\begin{gathered}
\left(\begin{array}{ccc}
12 \gamma_{1}+(1-10 i) \gamma_{2} & -25 \gamma_{2} & 12 \gamma_{1}+(1-10 i) \gamma_{2}+20 i \gamma_{2} \\
-12 \gamma_{2}+(1-10 i) \gamma_{1} & -25 \gamma_{1} & -12 \gamma_{2}+(1-10 i) \gamma_{2}+20 i \gamma_{1}
\end{array}\right), \\
v_{J}=\frac{v+\bar{v} J_{3}}{2}=9\left(\gamma_{1}^{2}+\gamma_{2}^{2}\right)^{-1}
\end{gathered}
$$

Then, we have $m=n=3, r=l=2$ and:

$$
\begin{array}{ll}
p=1, & L=\left(\begin{array}{lll}
0 & 1 & 0
\end{array}\right), \quad L^{\prime \prime}=1 \\
d=2, & Z=\left(\begin{array}{ll}
0 & 1 \\
0 & 0 \\
1 & 0
\end{array}\right), \quad R=\left(\begin{array}{ll}
0 & 1 \\
1 & 0
\end{array}\right) .
\end{array}
$$

We have $\mathcal{R}=\left\{\psi \in \mathbb{k}^{2 \times 1} \mid R \bar{\psi}=\psi\right\}$. Hence, if we note

$$
H=\left(\begin{array}{cccc}
0 & 1 & 0 & 0 \\
1 & 0 & 0 & 0 \\
0 & 0 & 0 & -1 \\
0 & 0 & -1 & 0
\end{array}\right) \in \mathbb{R}^{4 \times 4}
$$

then the eigenvalues of $H$ are -1 and 1 , and the eigenspace associated with 1 is defined by $G=\left(\begin{array}{ll}G_{1}^{T} & G_{2}^{T}\end{array}\right)^{T}$, where:

$$
G_{1}=\left(\begin{array}{ll}
0 & 1 \\
0 & 1
\end{array}\right), G_{2}=\left(\begin{array}{cc}
-1 & 0 \\
1 & 0
\end{array}\right) \text {. }
$$

Hence, we obtain:

$$
Z^{\prime}=G_{1}+i G_{2}=\left(\begin{array}{cc}
-i & 1 \\
i & 1
\end{array}\right), \quad \mathcal{R}=Z^{\prime} \mathbb{R}^{2 \times 1} .
$$

Since $l=\operatorname{rank}_{\mathfrak{k}}(M)=2$, we can now consider

$$
X=\left(\begin{array}{cc}
9+18 i & -225 \\
0 & 0 \\
9-198 i & -225
\end{array}\right), \quad Y=\left(\begin{array}{ccc}
1 & 0 & 1 \\
0 & 1 & -\frac{4}{5} i
\end{array}\right)
$$

which yields:

$$
\begin{gathered}
W_{1}=\left(\begin{array}{cc}
\frac{i}{216} & -\frac{i}{216} \\
-\frac{1}{2700}+\frac{i}{5400} & -\frac{11}{2700}-\frac{i}{5400}
\end{array}\right), \\
W_{2}=\left(\begin{array}{cc}
-\frac{1}{216} & -\frac{1}{216} \\
-\frac{1}{5400}-\frac{i}{2700} & -\frac{1}{5400}+\frac{11 i}{2700}
\end{array}\right) .
\end{gathered}
$$

For $\gamma \in \mathbb{R}^{2 \times 1}$, up to constant, the determinant of the matrix

$$
K(\gamma)=\left(W_{1} Z^{\prime} \gamma \quad W_{2} Z^{\prime} \gamma\right) \in \mathbb{k}^{2 \times 2}
$$

is $\gamma_{1}^{2}+\gamma_{2}^{2}$. Hence, if $\gamma_{1} \neq 0$ or $\gamma_{2} \neq 0$, then the centrohermitian rank factorization problem admits solutions. For $\gamma \in \mathbb{R}^{2 \times 1} \backslash\{0\}$, solutions $\left(u_{J}, v_{J}\right)$ are then defined by:$$
\left(\begin{array}{ccc}
12 \gamma_{1}+(1-10 i) \gamma_{2} & -25 \gamma_{2} & 12 \gamma_{1}+(1+10 i) \gamma_{2} \\
-12 \gamma_{2}+(1-10 i) \gamma_{1} & -25 \gamma_{1} & -12 \gamma_{2}+(1+10 i) \gamma_{1}
\end{array}\right) \text {. }
$$

Finally, if $d=1$ and $\mathcal{P} \neq \emptyset$, then we can easily prove that the centrohermitian rank factorization problem is solvable.

\section{REFERENCES}

[1] C. Capdessus. Aide au diagnostic des machines tournantes par traitement du signal. PhD thesis, University of Grenoble, France, 1992, https://hal.archives-ouvertes.fr/tel-02187906.

[2] C. Capdessus, M. Sidahmed. Analyse des vibrations d'un engrenage : cepstre, corrélation, spectre. Colloque francophone de traitement du signal et des images, Saint Martin d'Hères, vol. 8, 365-372, 1991.

[3] R. Dagher, E. Hubert, A. Quadrat. On the general solutions of a rank factorization problem arising in vibration analysis. Submitted.

[4] D. Eisenbud Commutative Algebra with a View Toward Algebraic Geometry. Springer, 1995.

[5] R. D. Hill, R. G. Bates, S. R. Waters. On Centrohermitian matrices. SIAM J. on Matrix Analysis and Applications, 11 (1990), 128-133.

[6] R. A. Horn, C. R. Johnson. Matrix Analysis. Cambridge University Press, 1985.

[7] R. A. Horn, D. I. Merino. A real-coninvolutory analog of the polar decomposition. Linear and Its Applications, 190 (1993), 209-227.

[8] E. Hubert. Amplitude and phase demodulation of multi-carrier signals: Application to gear vibration signals. PhD thesis, University of Lyon, France, 2019, https://tel.archives-ouvertes.fr/tel-02493898/.

[9] E. Hubert, A. Barrau, M. El Badaoui. New multi-carrier demodulation method applied to gearbox vibration analysis. Proceedings of ICASSP 2018, Calgary (Canada), 15-20/04/2018.

[10] E. Hubert, Y. Bouzidi, R. Dagher, A. Barrau, A. Quadrat. Algebraic aspects of the exact signal demodulation problem. Proceedings of SSSC \& TDS, Sinaia (Romania), 09-11/09/2019.

[11] E. Hubert, A. Barrau, Y. Bouzidi, R. Dagher, A. Quadrat. On a rank factorisation problem arising in gearbox vibration analysis. Proceedings of the 21st IFAC World Congress, Germany, 12-17/07/2020.

[12] E. Hubert, Y. Bouzidi, R. Dagher, A. Quadrat. Algebraic aspects of a rank factorization problem arising in vibration analysis. Proceedings of the Maple Conference, 02-06/11/2020.

[13] E. Hubert, Y. Bouzidi, R. Dagher, A. Quadrat. Centrohermitian solutions of a factorization problem arising in vibration analysis. Part I. Lee's transformation. Proceedings of ECC 2021.

[14] A. Lee. Centrohermitian and skew-centrohermitian matrices. Linear Algebra and its Applications, 29 (1980), 205-210.

[15] W. D. Mark. Analysis of the vibratory excitation of gear systems: Basic theory. The Journal of the Acoustical Society of America, 63 (1978), 1409-1430. 